\title{
Exit basins for the Sitnikov problem with variable mass
}

\author{
Cuencas de salida para el problema de Sitnikov con masa variable
}

\author{
E. A. Acosta-Pinzon iD ; F. L. Dubeibe iD; G. A. González iD
}

\begin{abstract}
In the present paper, we perform a numerical study of the Sitnikov problem aiming to characterize the orbits of a variable mass particle (e.g., comet, rocket, asteroid or spacecraft) and determine the uncertainty in the prediction of the final state of the test particle. The classification of final states was done through the well-known exit basins, while the determination of the uncertainty was calculated using a new tool named Basin entropy. It is found that for small values of the initial mass of the test particle, the number of initial conditions leading to bounded orbits gets increased, thus reducing the uncertainty in the final states. The same behavior in uncertainty is observed for increasing values of the exponent in Jeans law for the variation of the mass. Our results allow us to conclude that: i) an accelerated fuel consumption in the initial stages of stabilization of a satellite can keep the object in an oscillatory state around the primaries and ii) if the mass of the satellite is less than one hundredth of the mass of each primary, it is possible to predict with a very high certainty the final state of the satellite, regardless of the accuracy in the initial conditions of the system.
\end{abstract}

Index Terms - Basin entropy; Sitnikov problem; Variable mass, Jeans law

Resumen-En el presente trabajo, se realiza un estudio numérico del problema de Sitnikov que busca caracterizar las órbitas de una partícula de masa variable (e.g. un cometa, un cohete, un asteroide o una nave espacial), y determinar la incertidumbre en la predicción de los estados finales del sistema. La clasificación de los estados finales se realiza a través de las conocidas cuencas de salida, mientras que la determinación de la incertidumbre se calcula usando una nueva herramienta denominada entropía de las cuencas. Se encuentra que, para valores pequeños de la masa inicial de la partícula de prueba, el número de condiciones iniciales conducentes a órbitas acotadas aumenta significativamente, reduciendo así la incertidumbre en los estados finales. El mismo comportamiento en la incertidumbre se observa para valores grandes del exponente en la Ley de Jeans para la variación de la masa. Nuestros resultados permiten concluir que: i) un acelerado consumo de combustible en las etapas iniciales de

This manuscript was sent on May 15, 2019 and accepted on March 11, 2020. This work was supported by Colciencias under Grants No. 8840 and 8863.

E. A. Acosta-Pinzon is a PhD student of Physics, Universidad Industrial de Santander, Bucaramanga, Colombia. (e-mail: edgaracosta888@gmail.com).

F. L. Dubeibe is with the Faculty of Human Sciences and Education, Universidad de los Llanos, Villavicencio, Colombia. (e-mail: fdubeibe@unillanos.edu.co).

G. A. González is now with the Department of Physics, Universidad Industrial de Santander, Bucaramanga, Colombia. (e-mail: guillermo.gonzalez@saber.uis.edu.co). estabilización de un satélite puede mantener el objeto en un estado oscilatorio en torno a las primarias y ii) si la masa del satélite es menor a una centésima parte de la masa de cada primaria, es posible predecir con una altísima certidumbre el estado final del mismo, sin importar la exactitud en las condiciones iniciales del sistema.

Palabras claves - Entropía de cuencas; Problema de Sitnikov; Masa variable; Ley de Jeans.

Resumo-No presente trabalho, realizamos um estudo numérico do problema Sitnikov com o objetivo de caracterizar as órbitas de uma partícula de massa variável (por exemplo, cometa, foguete, asteróide ou espaçonave) e determinar a incerteza na previsão do estado final da partícula de teste . A classificação dos estados finais foi feita através das conhecidas bacias de saída, enquanto a determinação da incerteza foi calculada usando uma nova ferramenta chamada entropia da bacia. Verificou-se que, para pequenos valores da massa inicial da partícula de teste, o número de condições iniciais que levam a órbitas limitadas aumenta, reduzindo assim a incerteza nos estados finais. $O$ mesmo comportamento na incerteza é observado para valores crescentes do expoente na lei de Jeans para a variação da massa. Nossos resultados permitem concluir que: i) um consumo acelerado de combustível nos estágios iniciais de estabilização de um satélite pode manter o objeto em um estado oscilatório em torno das primárias e ii) se a massa do satélite for menor que um centésimo da massa de cada primário, é possível prever com uma certeza muito alta o estado final do satélite, independentemente da precisão nas condições iniciais do sistema.

Palavras-chave - Entropia de bacias; Problema de Sitnikov; massa variável; lei de jeans.

\section{INTRODUCTION}

A nalyzing the dynamics of a gravitational system has given the possibility of accurately predicting gravitational events such as the collision of two stars or the motions of the stars, moons, planets, satellites, and comets (see e.g. [1]). The theoretical framework to model the interaction of $n$ point masses moving under the influence of their mutual gravitational attraction is the so-called $n$-body problem. Here, it is known that for $n \geq 3$ there is no general analytical solution and hence several simplifications must be introduced, for example, the restricted $n$-body problems [2].

In the restricted three-body problem (hereafter RTBP) the primaries move in circular or elliptic trajectories (solutions of 
a two-body problem), while the mass of the third body is negligible in comparison with the masses of the primaries [3]. Since the formulation of the RTBP, it was proved by Lagrange that this system possesses five equilibrium points, nowadays named Lagrangian points [4]. Since then, the existence of Trojan asteroids near the Lagrangian points L4 and L5 of the Sun-Jupiter system [5], significantly increased the interest of the scientific community on this problem [6].

On the other hand, it is a well-known fact that many realistic systems vary their mass during the evolution of the system [7], e.g., comets lose mass with each passage near the Sun and satellites moving around a star surrounded by a cloud of dust vary their mass due to friction. In a seminal paper, Shrivastava \& Ishwar derived the equations of motion for the RTBP with variable mass, showing that the system of equations is equivalent to the RTBP with perturbing forces [8]. Despite this important step in the field of astrodynamics, the true dynamical nature of the system has been evaded for decades, and therefore, the dynamics of this particular system has never been considered in the literature.

In this paper, we shall analyze the Sitnikov problem $[9,10]$, which corresponds to a simplified version of the RTBP, by assuming that the mass of the satellite decreases with respect to time according to the Jeans law [11]. The variation of the parameters inherent to the equation for mass loss rate, allow us to determine the exit basins for orbits escaping through the positive or negative $Z$-direction and their respective basin entropies. To our knowledge, this is the first time that this system is analyzed by performing a systematic classification of orbits in phase space.

The paper is organized as follows: section II deals with the mathematical formulation of the system. In section III, the numerical methodology used to classify the orbits is presented. We close this section showing the basin diagrams in phasespace associated with the exit basins of the system. Next, in section IV, we discuss the basin entropy related to the numerical classification carried out in the present study. The most important conclusions of our work are summarized in section $\mathrm{V}$.

\section{EQUATIONS OF MOTION}

In the Sitnikov problem, the test particle $m$ oscillates along an axis that is perpendicular to the plane of motion formed by the elliptic trajectories of two equal mass primaries $m_{1}=$ $m_{2}=M$. Given that the mass of the test particle $m$ is negligible in comparison with the mass of the primaries, $m_{3} \ll M$, it does not affect their motion.

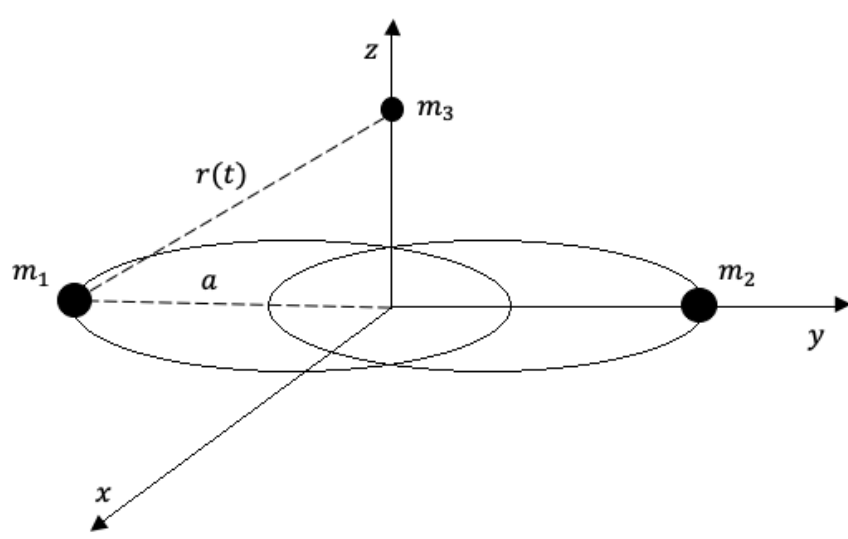

Fig. 1. The configuration of the Sitnikov problem. $r(t)$ denotes the distance of the center of mass to each primary, while $a$ is the semi-major axis of the ellipse.

Let us assume that the motion of the test particle (which varies its mass) takes place along the $z$-axis, as shown in Fig. 1. Under this condition, the Lagrangian for the Sitnikov problem reads as

$$
L=\frac{1}{2} m \dot{z}^{2}+\frac{2 G m M}{\sqrt{r(t)^{2}+z^{2}}}
$$

with $r(t)$ the distance of the center of mass to either primary

$$
r(t)=a(1-\varepsilon \cos t)+O\left(\varepsilon^{2}\right)
$$

while the equation of motion to the $\varepsilon^{2}$ order (see e.g. equation (3) in Ref. [9]) is given by

$$
m \ddot{z}+\dot{m} \dot{z}+\frac{2 G m M}{\left(z^{2}+a^{2}\right)^{3 / 2}}+\frac{6 G m M a^{2} z \varepsilon \cos t}{\left(z^{2}+a^{2}\right)^{5 / 2}}=0
$$

In all what follows we shall use standard units for the RTBP, i.e., the period of the primaries as $2 \pi$, the total mass of the primaries as mass unit $2 M=1$, the larger distance between the primaries as distance unit $2 a=1$, and the gravitational constants set as $G=1$. It should be noted that setting $\varepsilon=0$ in equation (3) we obtain the well know MacMillan problem [12].

On the other hand, based on the mass-luminosity relation, Jeans derived a law for the variation of mass in an astrophysical system [11]. In compact form, the equation for mass loss rate can be written as

$$
\dot{m}=-\alpha m^{n}
$$

with $\alpha$ a positive constant and $n$ an integer larger than 2 . Equation (1) can be analytically integrated to give the following expression for the mass as a function of time 


$$
m(t)=m_{0}\left[1+m_{0}{ }^{n-1} \alpha(n-1) t\right]^{1 /(1-n)}
$$

where $m_{0}$ is the initial mass at $t=0$. Setting $n=2$ and $n=3$ in equation (5), the first and second law of Meshcherskii are obtained [13]. In Fig. 2, we present the variation of $m$ as a function of time. Here, it can be seen that for larger values of $n$, the mass decays faster in time.

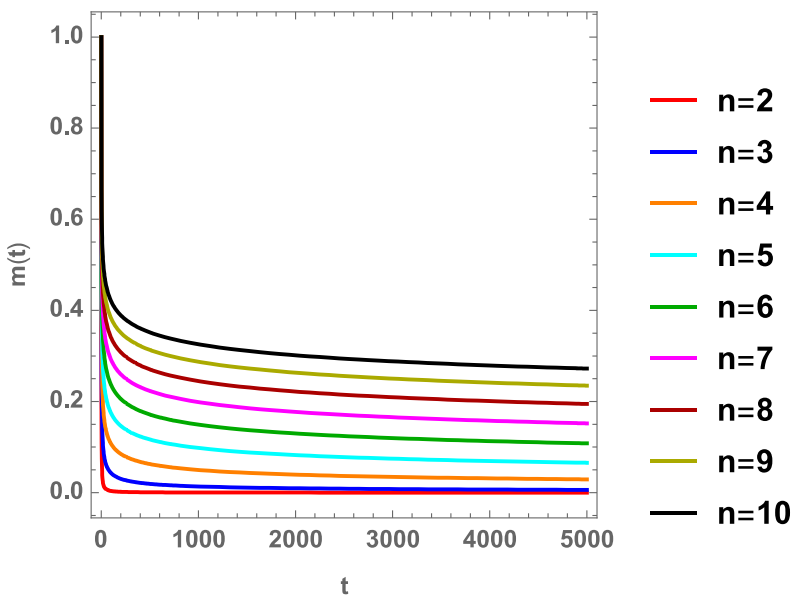

Fig. 2. Solutions for the mass as a function of time in the Jeans law. In all cases, we assume $m_{0}=1$ and $\alpha=2.7$.

After replacing equations (4) and (5) into equation (3), we obtain the explicit second-order differential equation for the Sitnikov problem with variable mass. This equation must be solved numerically and will depend on: the value of the parameter $n$ which characterizes the decay rate for the mass of the third body, the initial value of mass of the third body $m_{0}$, the parameter $\alpha$, and the eccentricity for the orbit of the primaries $\varepsilon$.

In order to check the dynamical behavior of the system, in

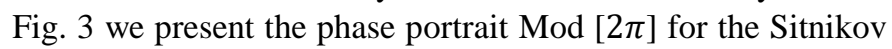
problem, under the approximation of low eccentricity orbits for the primaries. The chaotic behavior of the system is clearly observed when $\varepsilon>0$. In agreement with previous studies [14], the number of KAM islands are reduced for increasing values of the eccentricity. The interval of initial conditions was $z_{0}=[0,2.5]$ with $\Delta z_{0}=0.05$, and initial velocity $\dot{z}_{0}=$ 0 in all cases.

\section{EXIT BASINS}

It is a well-known fact that in a particular dynamical system with a single attractor or exit channel, the destination of any initial condition can be clearly and uniquely determined. However, the majority of dynamical systems often exhibit multistability, then, the determination of the set of orbits that tend to a specific attractor becomes a fundamental question. The study of these basins can provide information about the system since its topology is related to the dynamical nature of the system.

For an open system, we can define as exit basin the set of initial conditions escaping through some specific channel. As noted in the previous section the Sitnikov problem has three possible final states: exit $1(z \rightarrow \infty)$, exit $2(z \rightarrow-\infty)$, and non-escaping orbits. The criteria used to determine if a test particle escapes to one of the exit channels is that $|z| \geq 10^{4}$, because at that point the gravitational force has been reduced by eight orders of magnitude, making it practically negligible. According to the exit channel through which the test particle escapes, the initial condition is colored, i.e., escape through channel 1 with blue color, escape through channel 2 with red color, and the green regions denote initial conditions of nonescaping orbits.

In order to classify the orbits, we numerically solve the second-order differential equation for the Sitnikov problem with variable mass, by using a Runge-Kutta-Fehlberg Method (RKF45) implemented in $\mathrm{C}++$. This numerical code needed between 1 and 10 hours of CPU time on an Intel Xeon 3.0 $\mathrm{GHz}$ PC. Moreover, all graphical illustrations for the twodimensional color-coded diagrams have been created using the version 10.4.1 of the software Mathematica. 
(a)

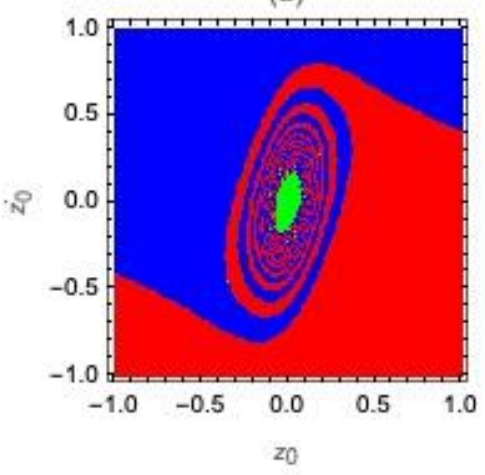

(d)

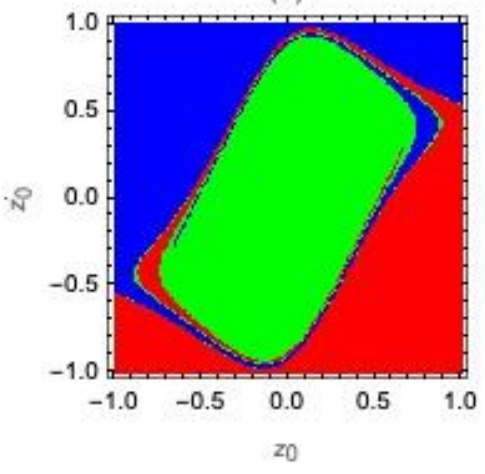

(g)

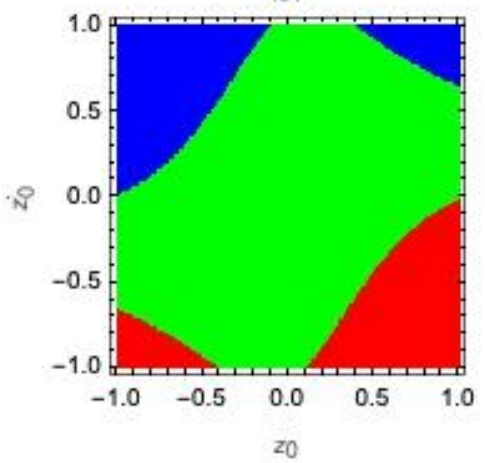

(b)

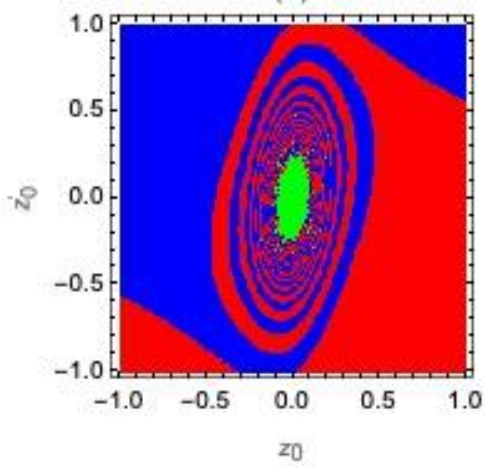

(e)

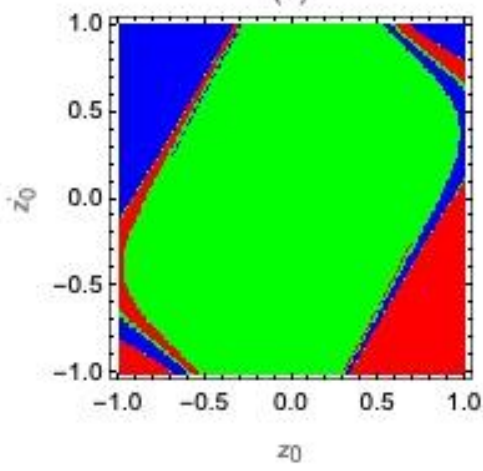

(h)

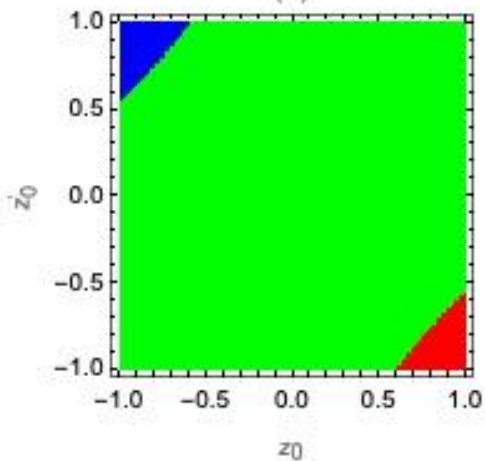

(c)

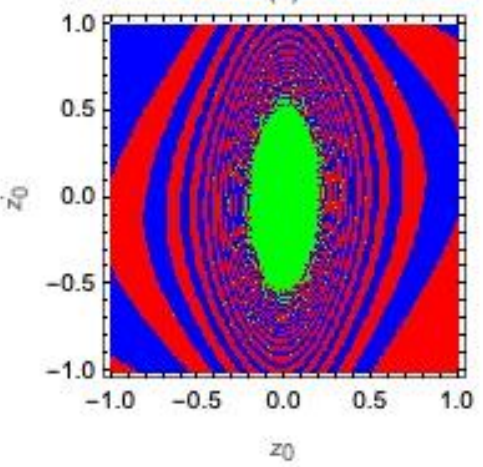

$(f)$

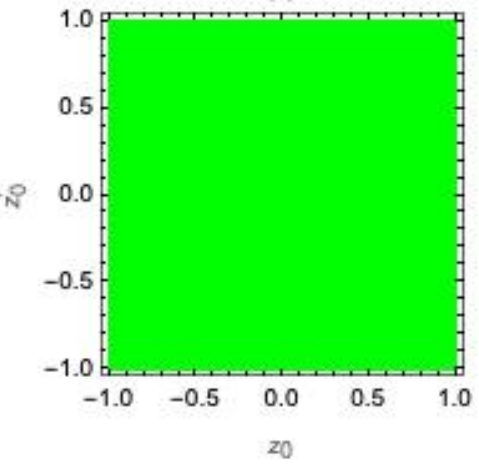

$(i)$

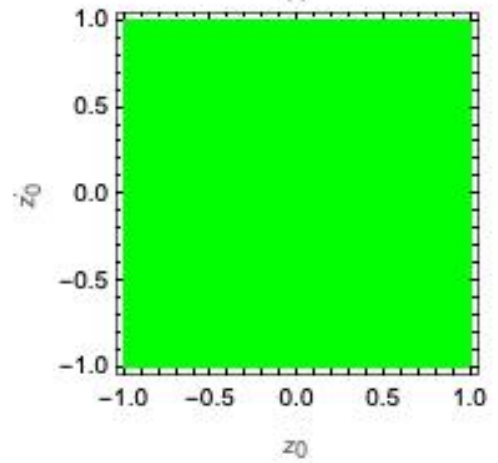

Fig. 4. Exit basins for the MacMillan problem using different values of the decay rate $n$ and the initial mass $m_{0}$. The first row corresponds to $n=2$, second row to $n=3$, and third row to the case $n=4$. Concerning the initial values of mass for the third body: the first column corresponds to $m_{0}=1$, second column to $m_{0}=0,5$, and the third column to $m_{0}=0.1$. In all cases, we set $\varepsilon=0$ and $\alpha=2.7$.

\section{A. Case $\varepsilon=0$}

Let us start considering the case in which the eccentricity of the primaries is zero, i.e., the MacMillan problem. The exit basins for the MacMillan problem $(\varepsilon=0)$ using different values of the decay rate $n$ and the initial mass $m_{0}$ are shown in Fig. 4. Here, it can be observed that the three possible final states take place. The first row of Fig. 4 corresponds to the situation in which $n=2$, panel (a) corresponds to $m_{0}=1$, panel (b) to $m_{0}=0.5$, and (c) to $m_{0}=0.1$. The second row corresponds to $n=3$, with $m_{0}=1$ in panel (d), $m_{0}=0.5$ in panel (e), and $m_{0}=0.1$ in panel (f). Finally, the third row depicts the case $n=4$, with $m_{0}=1$ in panel $(\mathrm{g}), m_{0}=0.5$ in panel (h), and $m_{0}=0.1$ in panel (i).
In the first row of Fig. 4, some complex structures of spiraltype are observed, these structures let us infer that despite the basins are smooth, the system becomes unpredictable when choosing a random initial condition belonging to the region of phase space $\left[-1 \leq z_{0} \leq 1\right]$ and $\left[-1 \leq \dot{z}_{0} \leq 1\right]$. It is also observed that when the initial mass decreased in magnitude, the basin corresponding to non-escaping orbits decreases.

In the case $n=3$ (panels (d), (e) and (f)), again, complex structures are present, and the region that characterizes bounded orbits grows as the initial mass of the test particle decreases. The main difference with the previous case is that the region related to non-escaping orbits, not only is larger for 
(a)

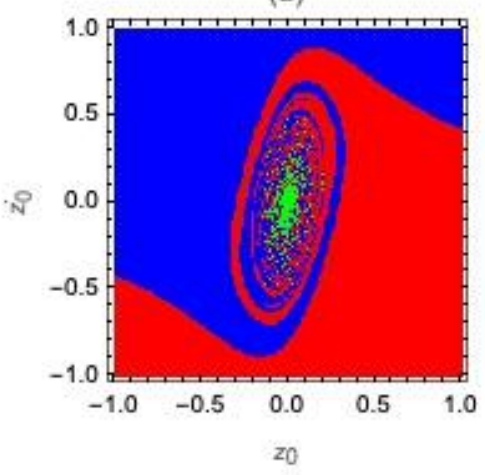

(d)

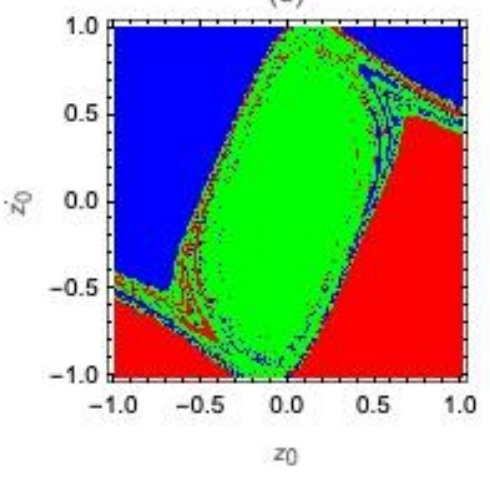

(g)

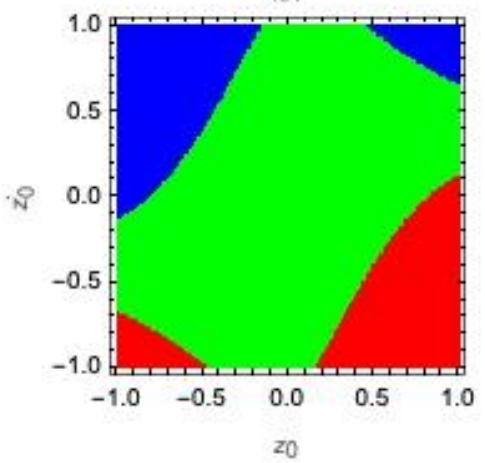

(b)

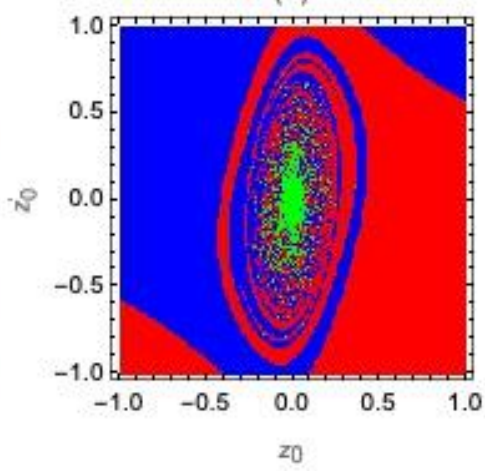

(e)

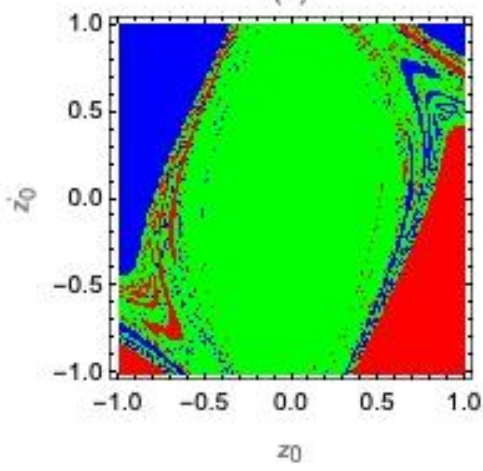

(h)

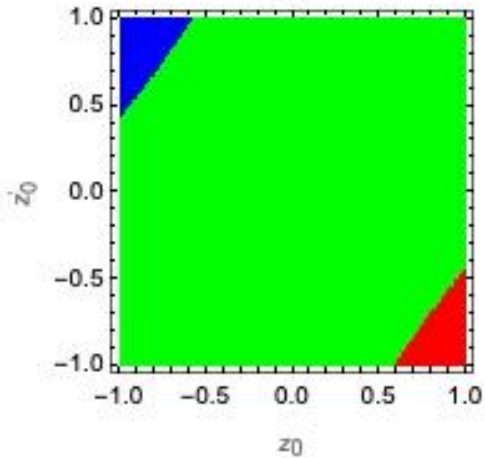

(c)

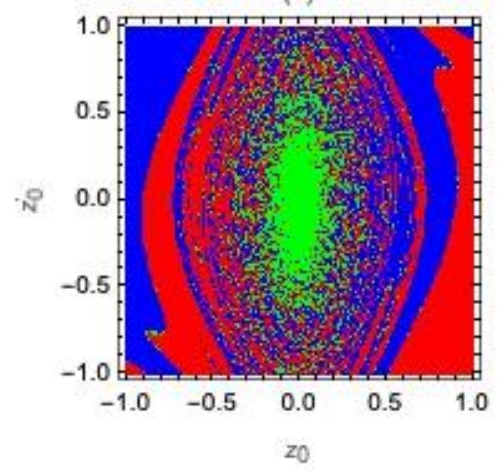

$(f)$

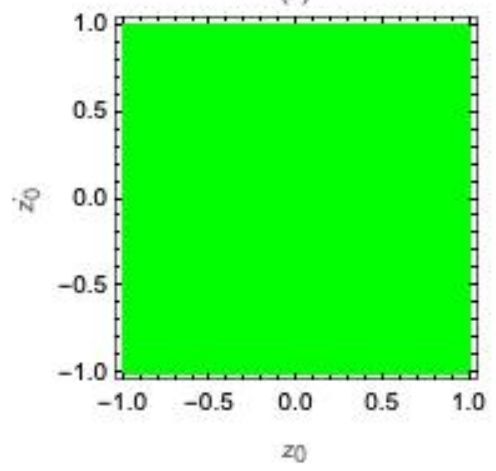

(i)

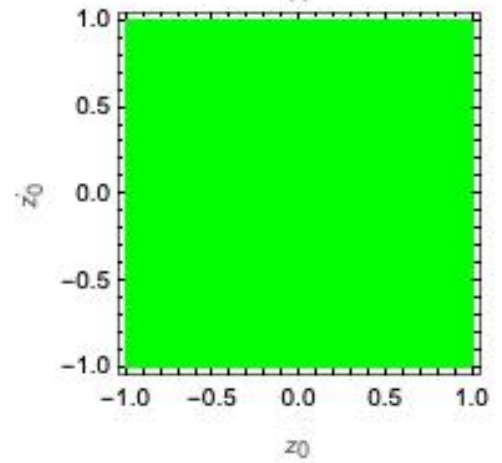

Fig. 5. Exit basins for the Sitnikov problem using different values of the decay rate $n$ and the initial mass $m_{0}$. The first row corresponds to $n=2$, second row to $n=3$, and third row to the case $n=4$. Concerning the initial values of mass for the third body: the first column corresponds to $m_{0}=1$, second column to $m_{0}=0,5$, and the third column to $m_{0}=0.1$. In all cases, we set $\varepsilon=0.1$ and $\alpha=2.7$.

$m_{0}=1$ in comparison to the previous case $(n=3)$, but also grows considerably faster.

For the group of basins corresponding to $n=4$, panels (g), (h) and (i) of the third row, the complex structures that were observed for $n=2$ and $n=3$ are no longer present, on the contrary, the basins correspond to well-defined smooth regions. Due to the fact that the area occupied by the green region is significantly larger than in the previous cases, the region of phase space $\left[-1 \leq z_{0} \leq 1\right]$ and $\left[-1 \leq \dot{z_{0}} \leq 1\right]$, gets almost totally filled with non-escaping orbits for small variations of the initial mass e.g. $m_{0}=0.5$.

\section{B. Case $\varepsilon=0.1$}

The second set of basins under consideration correspond to the case in which the eccentricity of the primaries is $\varepsilon=0.1$. As in the previous case, the first row of Fig. 5 depicts the situation in which $n=2$, second row $n=3$, and third row $n=4$. Each column is also associated to a value of the initial mass, i.e., panels (a), (d) and (g) correspond to $m_{0}=1$, panels (b), (e) and (h) to $m_{0}=0.5$, and panels (c), (f), and (i) to $m_{0}=0.1$. It is observed that the spiral-like structures seen in Fig. 4, also take place in this case, however, the boundaries of the green basins are dimmed. This result suggests that the chaotic nature of the Sitnikov problem is also reflected in the shape of the basins. The basins corresponding to $n=4$ are practically the same observed in the third row of Fig. 4. Also, 
(a)

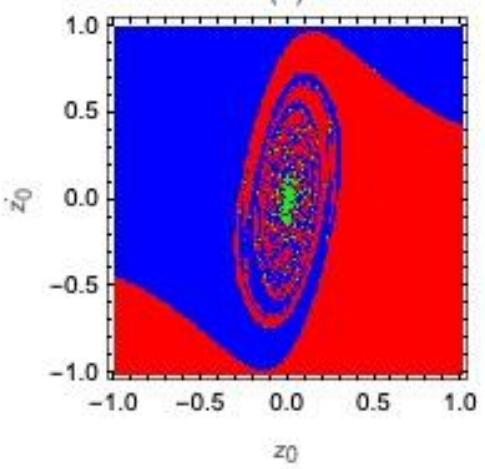

(d)

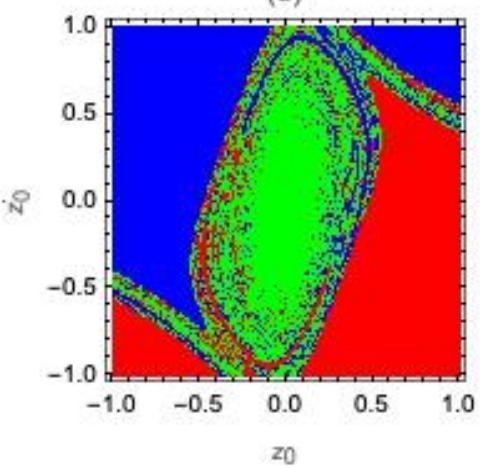

(g)

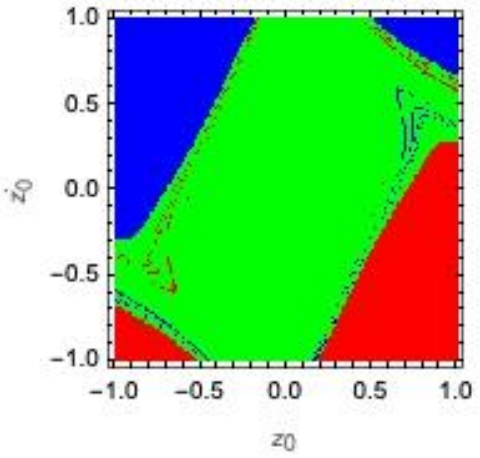

(b)

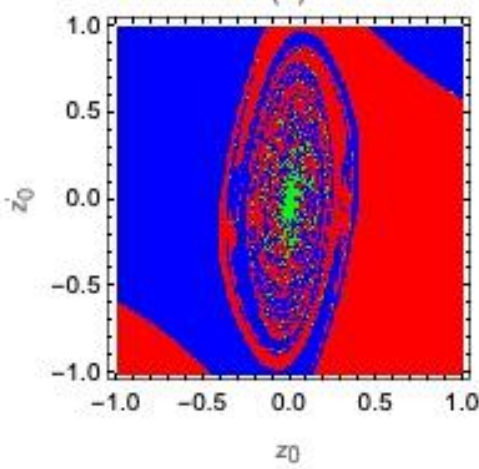

(e)

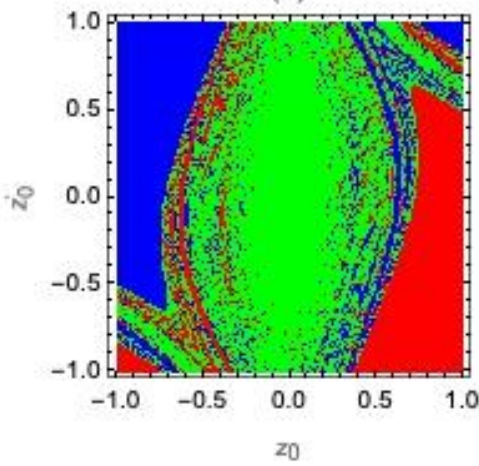

(h)

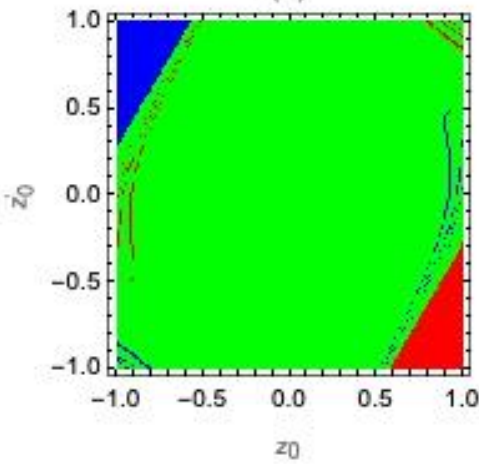

(c)

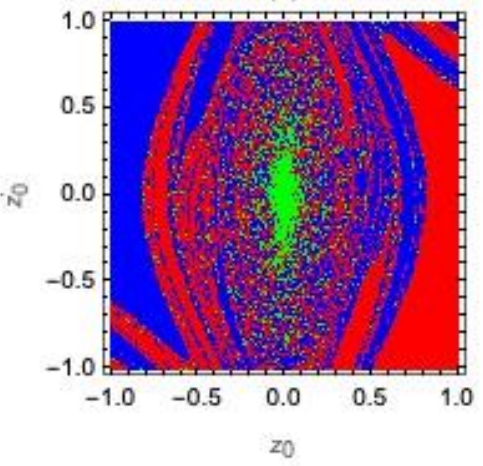

(f)

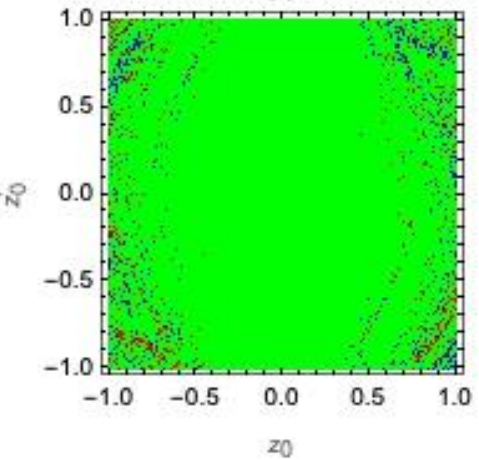

$(i)$

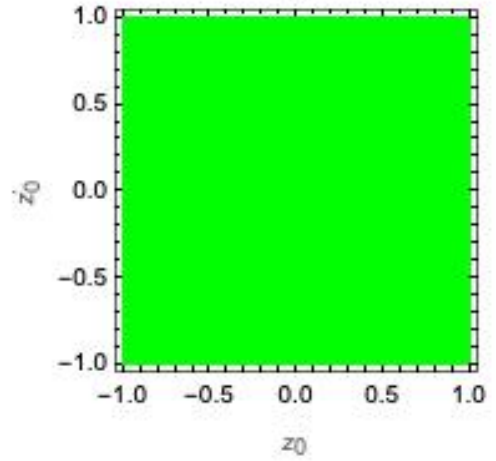

Fig. 6. Exit basins for the Sitnikov problem using different values of the decay rate $n$ and the initial mass $m_{0}$. The first row corresponds to $n=2$, second row corresponds to $n=3$, and third row to the case $n=4$. Concerning the initial values of mass for the third body: the first column corresponds to $m_{0}=1$, second column to $m_{0}=0,5$, and the third column to $m_{0}=0.1$. In all cases, we set $\varepsilon=0.2$ and $\alpha=2.7$.

it should be noted that a small value of the initial mass $m_{0}<$ 0.1 , along with a value of the decay rate larger than 2 , are enough to fill the phase space with bounded orbits.

\section{Case $\varepsilon=0.2$}

The last case under consideration refers to a larger eccentricity value $\varepsilon=0.2$ (see Fig. 6). The ergodic distribution of the initial conditions tending to a particular basin becomes more marked. For $n=2$, it is observed that the spirals are destroyed independently of the initial mass value. Also, it can be noted that the dense green region associated with bounded orbits is reduced, but at the same time, there are some random initial conditions distributed along with the available phase space. Panels (d) and (e) in Fig. 6 are similar to the ones presented in Fig. 5, however the main difference is related to the basins of panels (f), where the highest value of eccentricity in Fig. 6 erases the smooth and uniform distribution on the basin of bounded orbit, allowing the possibility to obtain final states of the exits 1 and 2 .

In the last row of Fig. 6, panels (g) and (h), it is observed that some blue and red basins invaded the inner green region, such that the uncertainty about the final states is also increased. Finally, as for the distribution of basins shown in panels (i) of Figs. 4 y 5, the last panel of Fig. 6 exhibits a homogeneous filling of the phase space.

\section{BASIN ENTROPY}

The concept of basin entropy is a new quantitative measure 
recently introduced to determine the uncertainty of a given basin [15]. In this context, the term uncertainty can be understood as the difficulty to easily predict the final state of a given set of initial conditions. The methodology to calculate the basin entropy consists of subdividing the phase space into $N$ small cells, such that each cell must include at least one final states $N_{A}$. The entropy associated with the cell $i$, is given by the expression

$$
S_{i}=\sum_{j=1}^{m_{i}} p_{i, j} \log \left(\frac{1}{p_{i, j}}\right)
$$

where $p_{i, j}$ denotes the probability to find a state $j$ in the cell $i$, and $m_{i} \in\left[1, N_{A}\right]$ represents the number of final states inside the $i$-th box.

The total entropy for the basin $S_{b}$, is then calculated as the sum of individual entropies normalized by the total number of boxes $N$, i.e.,

$$
S_{b}=\frac{1}{N} \sum_{i=1}^{N} \sum_{j=1}^{m_{i}} p_{i, j} \log \left(\frac{1}{p_{i, j}}\right)
$$

At this point, it is important to point out that the basin entropy $S_{b}$ directly depends on the total number of boxes of the basin. To avoid this limitation, we use a Monte Carlo procedure to randomly select the boxes inside the basin. In the present paper, following the suggestion presented in Ref. [16] we use $\epsilon=5$, for the size of the boxes and $N=1 \times 10^{5}$ for the total number of boxes, aiming to get a constant and realistic value for $S_{b}$.

TABLE I

BASIN ENTROPY FOR $\varepsilon=0$

\begin{tabular}{cccc}
\hline \hline & $m_{0}=1.0$ & $m_{0}=0.5$ & $m_{0}=0.1$ \\
\hline$n=2$ & 0.12 & 0.18 & 0.36 \\
$n=3$ & 0.07 & 0.05 & 0.0 \\
$n=4$ & 0.01 & $4 \times 10^{-3}$ & 0.0 \\
\hline \hline
\end{tabular}

In Tables 1, 2, and 3, we present the resulting values for the basin entropy of Figs. 4, 5, and 6. From Table I, it can be noted that in the MacMillan problem $(\varepsilon=0)$ the largest value of the basin entropy corresponds to the panel (c) of Fig. 4 . This result can be explained by considering that this plot does not contain large zones of red, blue or green basins, in other words, the number of basin boundaries is larger than in the other panels. The same result is observed for $\varepsilon=0.1$ and $\varepsilon=$ 0.2 , in Tables II and III, respectively. In the same vein, the smaller values of the basin entropy correspond to panels (i), because in this case all the diagrams only possess one final state.
TABLE II

BASIN ENTROPY FOR $\varepsilon=0.1$

\begin{tabular}{lccc}
\hline \hline & $m_{0}=1.0$ & $m_{0}=0.5$ & $m_{0}=0.1$ \\
\hline$n=2$ & 0.12 & 0.19 & 0.42 \\
$n=3$ & 0.12 & 0.13 & 0.00 \\
$n=4$ & 0.01 & $4 \times 10^{-3}$ & 0.00 \\
\hline \hline
\end{tabular}

As a general result, it can be noted that the increase in the eccentricity of the primaries also increases the values of the basins entropies, for example panels (g) of Figs. 4 and 5 have a zero value for the entropy, but for the case $\varepsilon=0.2$, this value is about 0.1 .

TABLE III

BASIN ENTROPY FOR $\varepsilon=0.2$

\begin{tabular}{lccc}
\hline \multicolumn{4}{c}{ BASIN ENTROPY FOR $\varepsilon=0.2$} \\
& $m_{0}=1.0$ & $m_{0}=0.5$ & $m_{0}=0.1$ \\
\hline$n=2$ & 0.13 & 0.20 & 0.46 \\
$n=3$ & 0.21 & 0.28 & 0.10 \\
$n=4$ & 0.04 & 0.03 & 0.00 \\
\hline \hline
\end{tabular}

\section{CONCLUSIONS}

The scope of this paper was to investigate the basins of escape or exit basins of the Sitnikov problem with variable mass. For describing the variation of mass of the third mass, we used the Jeans law, which reduces to the first and second law of Meshcherskii for $n=2$ and $n=3$, respectively. The resulting autonomous second-order differential equation was numerically integrated for a large set of initial conditions in the region of phase space $\left[-1 \leq z_{0} \leq 1\right]$ and $\left[-1 \leq \dot{z}_{0} \leq 1\right]$. By means of color-coded basin diagrams and with the aid of the basin entropy, we demonstrate the influence of the eccentricity in the final states of the orbits as well as in the degree of fractality of the system. The main results of our study can be summarized as follows:

- It is found that at a lower value of the initial mass the number of initial conditions that end up in bounded orbits is greater.

- The fractal degree of the system is increased with increasing value of $\varepsilon$, since all the basin boundaries become less smooth, and at the same time, all the noisy (chaotic) regions are amplified. The increment in the fractality was confirmed by the computation of the basin entropy.

- In all cases, it is found that by increasing the value of the mass loss rate, the value of the basin entropy tends to be reduced. Then, it can be conjectured that a fast drop on the mass of the satellite should decrease the uncertainty in the determination of its final state.

- The results indicate that a residual character of the 
chaotic dynamics of bounded orbits, is reflected in the increase of the uncertainty of basins for unbounded orbits.

- Our findings are in agreement with previous studies related to the influence of perturbing terms in the dynamics of open and closed astrophysical systems $[17,18,19]$.

\section{ACKNOWLEDGMENT}

This research was partially supported by VIE-UIS, under grant number 2416, and COLCIENCIAS, Colombia, under grant numbers 8840 and 8863 .

\section{REFERENCES}

[1] B. G. Marchand, K. C. Howell and R. S. Wilson, "Improved corrections process for constrained trajectory design in the n-body problem," Journal of Spacecraft and Rockets, vol. 44, no. 4, pp. 884-897, 2007. DOI: $10.2514 / 1.27205$

[2] K. Meyer, G. Hall and D. Offin, Introduction to Hamiltonian dynamical systems and the N-body problem, Springer Science \& Business Media, 2008. DOI: $10.1137 / 1035155$

[3] C. Marchal, The three-body problem, Elsevier, 2012.

[4] M. Hénon, Generating families in the restricted three-body problem, vol. 52, Springer Science \& Business Media, 2003.

[5] C. Murray and D. Stanley, Solar system dynamics, Cambridge: Cambridge university press, 1999.

[6] A. Morbidelli, H. F. Levison, K. Tsiganis and R. Gomes, "Chaotic capture of Jupiter's Trojan asteroids in the early Solar System," Nature, vol. 435, no. 7041, p. 462, 2005. DOI: 10.1038/nature03540

[7] M. Colpi, M. Lucio and F. Governato, "Dynamical friction and the evolution of satellites in virialized halos: the theory of linear response," The Astrophysical Journal, vol. 525, no. 2, p. 720, 1999. DOI: $10.1086 / 307952$

[8] A. K. Shrivastava and B. Ishwar , "Equations of motion of the restricted problem of three bodies with variable mass," Celestial mechanics, vol. 30, no. 3, pp. 323-328, 1983. DOI: 10.1007/BF01232197

[9] J. Liu and Y. S. Sun, "On the Sitnikov problem," Celestial Mechanics and Dynamical Astronomy, vol. 49, p. 285-302, 1990. DOI: 10.1007/BF00049419

[10] K. Sitnikov, "The existence of oscillatory motions in the three-body problem," Dokl. Akad. Nauk SSSR, vol. 133, no. 2, pp. 303-306, 1960.

[11] J. H. Jeans, "Cosmogonic problems associated with a secular decrease of mass," Monthly Notices of the Royal Astronomical Society, vol. 85, p. 2, 1924. DOI: $10.1093 / \mathrm{mnras} / 85.1 .2$

[12] W. D. MacMillan, "An integrable case in the restricted problem of three bodies," The Astronomical Journal, vol. 27, pp. 11-13, 1911. DOI: 10.1086/103918

[13] V. Meshcherskii, Studies of the Mechanics of Bodies of Variable Mass, Moscow: GITTL, 1952.

[14] T. Kovács and B. Érdi, "Transient chaos in the Sitnikov problem," Celestial Mechanics and Dynamical Astronomy, vol. 105, no. 4, p. 289, 2009. DOI: $10.1007 / \mathrm{s} 10569-009-9227-0$

[15] A. Daza, A. Wagemakers, B. Georgeot, D. Guéry-Odelin and M. A. Sanjuán, "Basin entropy: a new tool to analyze uncertainty in dynamical systems," Scientific reports, vol. 6, p. 31416, 2016. DOI: 10.1038/srep31416

[16] A. Daza, B. Georgeot, D. Guéry-Odelin, A. Wagemakers and M. A. Sanjuán, "Chaotic dynamics and fractal structures in experiments with cold atoms," Physical Review A, vol. 95, no. 1, p. 013629, 2017. DOI: 10.1103/PhysRevA.95.013629

[17] F. L. Dubeibe, F. D. Lora-Clavijo and G. A. González, "PseudoNewtonian planar circular restricted 3-body problem," Physics Letters A, vol. 381, no. 6, pp. 563-567, 2017. DOI: 10.1016/j.physleta.2016.12.024
[18] E. Zotos and F. L. Dubeibe, "Orbital dynamics in the post-Newtonian planar circular restricted Sun-Jupiter system," International Journal of Modern Physics D, vol. 27, no. 4, p. 1850036, 2018. DOI: $10.1142 / \mathrm{S} 0218271818500360$

[19] F. L. Dubeibe, A. Riaño-Doncel and E. E. Zotos, "Dynamical analysis of bounded and unbounded orbits in a generalized Hénon-Heiles system," Physics Letters A, vol. 382, no. 13, pp. 904-910, 2018. DOI: 10.1016/j.physleta.2018.02.001

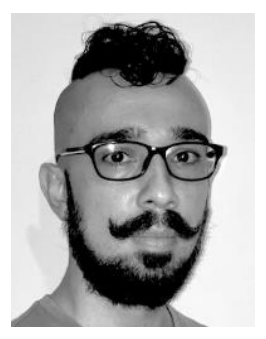

Edgar A. Acosta Pinzón, Magister en Matemática Aplicada, Físico. Estudiante del programa de Doctorado en Física de la Universidad Industrila de Santander. Miembro del grupo de investigacion en relatividad $\mathrm{y}$ gravitacion GIRG (UIS).

ORCID: https://orcid.org/0000-00025327-7316

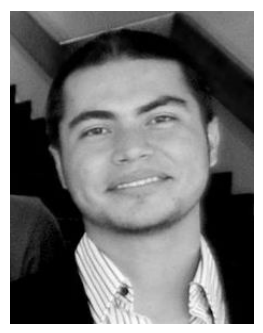

Fredy L. Dubeibe, Doctor en Ciencias: Física, Magister en Física, Físico. Docente de la Facultad de Ciencias Humanas y de la Educacion de la Universidad de los Llanos. Director del grupo de investigacion Cavendish (Unillanos).

ORCID: https://orcid.org/0000-00020793-3255

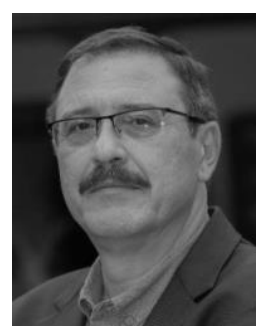

Guillermo A. González, Doctor en Matemática Aplicada, Magister en Física, Físico. Docente del Departamento de Física de la Universidad Industrial de Santander. Director del grupo de investigacion en relatividad y gravitacion GIRG (UIS).

ORCID: https://orcid.org/0000-00015805-9388 